Case report

\title{
The young breast cancer patient therapy - new opportunities, new challenges
}

\author{
Joanna Załuska-Kusz', Joanna Kufel-Grabowska ${ }^{1,2}$ \\ 'Department of Chemotherapy, Greater Poland Cancer Center \\ ${ }^{2}$ Department of Electroradiology, Poznań University of Medical Sciences
}

Correspondence:

Joanna Załuska-Kusz Greater Poland Centre of Oncology in Poznań 61-866 Poznań, Garbary 15 e-mail:joanna.zaluska@wco.pl

Received: 19.04.2019

Accepted:

9.05.2019

DOl: 10.24292/01.OR.219090519 Copyright ๑ Medical Education. All rights reserved.

\section{ABSTRACT}

The paper describes the case of a 31-year-old female patient with breast cancer with the abdominal metastases. This disease in young people usually has a more aggressive biological subtype, which significantly worsens the prognosis. In this patient aggressive treatment was used. It was effective and did not cause severe or long-term complications.

Key words: breast cancer in young patient, non-pegylated liposomal doxorubicin, ribociclib, metastatic disease 


\section{INTRODUCTION}

Breast cancer is the most common malignancy in women across the world, preceded only by lung cancer in terms of mortality. It is usually detected in post-menopausal women, which is why screening mammography testes are dedicated to female patients aged 50-69. Despite the observed increase in breast cancer incidence in young women, it is still rarely diagnosed in patients below the age of 40 . In that group of patients, gene mutations that increase the risk of breast and ovarian cancer, including $B R C A 1 / 2$, are more commonly found, affecting up to $20-25 \%$ of the younger population of female patients. Hence, each young patient should have access to genetic counseling. Young women who are not carriers of pathogenic mutations are not subject to preventative breast and ovarian check-ups, which is why breast cancer is often detected at a more advanced stage in them than in older patients. Clinical symptoms are often the first impulse to embark on a diagnostic process that often proves difficult due to the glandular structure of the breast, which furthers delays treatment.

In young women, breast cancer reveals a more aggressive biological subtype than in post-menopausal women, with triple negative cancer (without HER2 overexpression or gene amplification, and without hormone receptor expression in cancer cell nuclei) and HER2-positive cancer diagnosed more frequently in that group, which is associated with poorer prognosis.

The clinical case presented below is interesting for a number of reasons. It involves a young patient with positive family history, diagnosed with luminal cancer. Despite its intensity, the treatment applied did not cause severe or long-term complications in the patient. The slow course of the disease requires an effective therapy that will not comprise the patient's daily life at all or will only have a minimum impact on her day-to-day affairs.

\section{CASE DESCRIPTION}

In May 2016, a 31-year-old patient reported to an oncologist, having discovered a lump in her right breast. Breast ultrasound was performed at the time, revealing two tangentially located heterogeneous and hypoechogenic periareolar lesions in the upper outer quadrant of the right breast, with the largest cross-section of $30 \times 17 \mathrm{~mm}$, medially $27 \times 16 \mathrm{~mm}$; otherwise, there were no focal lesions within the breast. Abdominal CT revealed around 20 nodular lesions in the liver, whose diameter was ca. $20 \mathrm{~mm}$. The lesions were histologically confirmed to be breast cancer metastases. Remaining imaging tests (chest $x$-ray, bone scintigraphy) did not reveal other metastatic lesions. The patient's gener- al condition was very good, without comorbidities, and without symptoms related to the disease.

The patient's family history was positive, with the patient's mother diagnosed with breast cancer at the age of 48 . The patient was referred to a genetic clinic, but she was not found to be a carrier of pathogenic founder mutations in the BRCA1 gene.

Core biopsy of the right breast tumor was performed, revealing the NHG2 infiltrating ductal carcinoma; estrogen receptor expression was confirmed in over $75 \%$ of cancer cell nuclei, and progesterone receptor presence was revealed in $10-75 \%$ of cancer cell nuclei. HER2 was negative in the cells of the infiltrating carcinoma (Score $=1+$ ). Expression of the Ki-67 proliferative antigen was revealed in ca. $50 \%$ of the cell nuclei.

In May 2016, the patient was started on the AC chemotherapy regimen (doxorubicin $60 \mathrm{mg} / \mathrm{m}^{2}$, cyclophosphamide $600 \mathrm{mg} /$ $\mathrm{m}^{2}$ IV every 21 days). The patient received 6 courses in total, with partial remission of the metastatic lesions in the liver, and partial remission of the breast tumor. Before the initiation of treatment, her CA 15.3 blood level was $174.5 \mathrm{U} / \mathrm{ml}$, and after the first course of chemotherapy it dropped to $26.8 \mathrm{U} / \mathrm{ml}$, later remaining within normal limits throughout the therapy (below $30 \mathrm{U} / \mathrm{ml}$ ). As the patient responded to the therapy, and tolerated it very well, treatment with non-pegylated liposomal doxorubicin dosed at $60 \mathrm{mg} / \mathrm{m}^{2}$ was continued. It lasted from September 2016 to May 2017, and involved 12 courses of chemotherapy, including the classical form of doxorubicin. The patient received all of the courses within the prescribed timeframe. There were no significant abnormalities in the lab tests performed, such as neutropenia, anemia or thrombocytopenia. Throughout the treatment, follow-up imaging tests were performed, including abdominal ultrasound and abdominal CT, repeated every 3 months. Each consecutive test revealed a further remission of the lesions involved. In July 2017, the patient had a PET scan that revealed 3 nodular lesions in the liver, whose size was below $1 \mathrm{~cm}$, and which did not show a pathologically increased FDG activity. There were no suspicious lesions within the breast tissue either.

During the anthracycline therapy, it turned out that the patient could be qualified for the EMBRACA clinical trial dedicated to patients with confirmed mutations in the BRCA1/2 genes, and to patients at risk of mutations. Hence, $B R C A 1 / 2$ gene sequencing was performed from the patient's blood sample, revealing no pathogenic mutations. Thus, the patient was not qualified for the study. 
In June 2017, the patient initiated hormone therapy with tamoxifen combined with an LHRH analogue.

The patient was in remission until August 2018, when a follow-up PET scan revealed disease progression. 2 foci were then revealed in the liver parenchyma, presenting metabolic features of active metastatic neoplastic lesions. There was also a suspicious focus of increased glucose utilization in the right breast, and a new ovarian lesion. Lab tests showed no abnormalities, with the Ca 15.3 marker within normal limits (below $30 \mathrm{U} / \mathrm{ml}$ ). The patient was referred to a gynecologist for further assessment of the detected ovarian lesion. She was qualified for adnexectomy. The right ovarian tumor was confirmed to be a breast cancer metastasis, showing expression of hormone receptors, without overexpression of the HER2 receptor.

In October, the patient was started on letrozole dosed at $2.5 \mathrm{mg} / 24 \mathrm{~h}$, administered continuously, in combination with ribociclib dosed at $600 \mathrm{mg} / 24 \mathrm{~h}$, administered for 21 days, and followed by a 7-day interval. So far, the patient has received 5 courses of ribociclib. ECG tests were regularly repeated during the treatment, initially every 2 weeks, and later on a monthly basis. QTcF interval was never prolonged beyond $440 \mathrm{~ms}$. The patient has tolerated the therapy well, leading a normal life, and remaining professionally active. Since the beginning of the treatment, CTCAE (Common Terminology Criteria for Adverse Events) grade II neutropenia has been observed, and there has been a single episode of neutrophil drop to $<1 \mathrm{G} /$, which was brought up to $>1.5 \mathrm{G} /$, following a 7-day delay in treatment.

Follow-up imaging tests, including chest $\mathrm{X}$-ray, abdominal ultrasound and bone scintigraphy, revealed 3 metastatic foci in the liver, which have gradually decreased in size. Other metastatic lesions have not been detected.

\section{DISCUSSION}

Doxorubicin is an anthracycline antibiotic with anti-cancer properties, and as such it is widely applied in the treatment of many different tumors. It is incorporated in the DNA structure, causing its disruption and fragmentation, thus leading to an inhibition of DNA replication. It also affects topoisomerase II, cell membranes, production of free radicals and apoptosis. Doxorubicin is used in the treatment of breast cancer, both in radical treatment as well as in metastatic disease, combined with other anti-cancer drugs or as stand-alone therapy.

Anthracycline-induced heart failure is one of the most common cardiovascular complications in patients undergoing chemo- therapy. Before initiating treatment, ECG and echocardiogram should be performed in each patient, with the assessment of the left ventricular ejection fraction. The cardiotoxic effect of doxorubicin is related to the chelation of metal ions, and iron in particular, which leads to the creation of coordination compounds with potent oxidative properties, and to the release of free radicals. Mitochondrial function is thus compromised, leading to reduced energy production, and compromised systolic and diastolic function of the heart. The risk of cardiac complications is increased, once the cumulative dose of $550 \mathrm{mg} / \mathrm{m}^{2}$ is reached. The dose is lower $\left(450 \mathrm{mg} / \mathrm{m}^{2}\right)$ in patients who have previously undergone mediastinal radiotherapy and/or in patients with concomitant diseases (e.g. arterial hypertension, diabetes, lipid disorders). Risk factors of cardiotoxicity also include combined chemotherapy (involving, for instance, taxanes or cyclophosphamide) and patient age below 15 or over 70 years of age. Anthracycline-induced cardiac injury is usually irreversible.

In patients who received the cumulative dose of doxorubicin, and benefitted from the therapy, a possible option for continued treatment with anthracyclines is to receive non-pegylated liposomal doxorubicin. A meta-analysis involving 10 clinical studies that looked into the efficacy and cardiac safety of the drug indicated that there was a statistically significantly reduced risk of cardiotoxicity, and an increased rate of objective response following the administration of that novel form of doxorubicin as compared with the conventional product. No differences have been reported as for the progression-free survival and overall survival.

\section{SUMMARY}

In the above discussed case, partial remission was accomplished both with respect to the breast tumor as well as the liver metastases during the treatment involving doxorubicin and cyclophosphamide. The patient received $360 \mathrm{mg} / \mathrm{m}^{2}$ (a total dose of $619 \mathrm{mg}$ ) of doxorubicin. Due to the high risk of cardiotoxicity related to continued treatment, it was decided that non-pegylated liposomal doxorubicin dosed at $60 \mathrm{mg} / \mathrm{m}^{2}$ would be applied. The patient received 12 courses of chemotherapy, with further disease remission observed. ECG and echocardiogram tests were performed before the first course of treatment, and then every 3 months. Left ventricular ejection fraction was $68-70 \%$ for each consecutive test, and no abnormalities were found. The patient did not report any complaints related to the cardiovascular system. The PET scan performed after the last course of liposomal doxorubicin did not reveal any active neoplastic lesions (complete remission). The patient has tolerated the subsequent treatment lines very well, with good therapeutic outcomes. 


\section{References}

1. Batist G, Remakrishnan G, Rao CS et al. Reduced cardiotoxity and preserved antitumor efficacy of liposome-encapsulated doxorubicin and cyclophosphamide compared with conventional doxorubicin with cyclophosphamide in a randomized, multicenter trial of metastatic breast cancer. J Clin Oncol 2001; 19: 1444-54.

2. Batist G, Harris L, Azarina $\mathrm{N}$ et al. Improved anti-tumor response rate with decreased cardiotoxity of non-pegylated liposomal doxorubicin compared with conventional doxorubicin in first-line treatment of metastatic breast cancer in patients who haved received prior adjuvant doxorubicin: results of a retrospective analysys. Anticancer Drugs 2006; 17: 587-95.

3. Meiyuan X, Feifei Y, Sufen Y, Peng S. Efficacy and cardiotoxicity of liposomal doxorubicin-based chemotherapy in advanced breast cancer: a meta-analysis of ten randomized trials. PloS One 2015; 10(7): e0133569.

Authors' contributions: Joanna Załuska-Kusz: 50\%; Joanna Kufel-Grabowska: 50\% Conflict of interests: None.

Financial support: None. Ethics: 